\title{
NOTE ON A THEOREM OF BEURLING
}

\author{
MAX L. WEISS ${ }^{1}$
}

1. Introduction. In [3] Somadasa proved the following theorem:

THEOREM [3, p. 297]. Let $\mu$ be a fixed number greater than or equal to 1. Then, corresponding to each point $e^{i \theta}$ on the unit circle, $C$, in the complex plane, we can construct a class of Blaschke products with the property that each member of this class has $T_{\mu}$-limit zero at $e^{i \theta}$. Further, there exists a nonempty subclass of this class with the property that each member of this subclass has $T_{\mu}$-limit zero at $e^{i \theta}$ for all values of $\mu$.

The purpose of this note is to prove a (slightly stronger form of a) theorem (Theorem 1, below) of Beurling [4]. (No detailed proof of Beurling's Theorem exists in the literature.) This theorem in turn generalizes the result of Somadasa in two directions. First, the class of Blaschke products is replaced by a much larger class of bounded analytic functions. Second, the restriction to $T_{\mu}$-limits is replaced by essentially arbitrary approaches.

2. Beurling's Theorem. Let $D$ denote the open unit disc in the complex plane, $C$, the unit circle, $H^{\infty}$, the collection of all bounded analytic functions on $D$. The pseudo-hyperbolic metric, $\chi$, on $D$ is defined by $\chi(z, w)=|z-w| /|1-\bar{z} w|$. We recall two classical theorems from the theory of complex variables.

Pick's Theorem [1, p. 48]. Let $f \in H^{\infty}$, and suppose $f$ maps into $D$. Then for any two points $z, w \in D$ one has

$$
\chi(f(z), f(w)) \leqq \chi(z, w) .
$$

LINDELÖF'S THEOREM [1, p. 76]. Let $G$ be a region bounded by a simple closed curve $\Gamma$, let $p \in \Gamma$. Let $f$ be continuous on $(G \cup \Gamma)-\{p\}$, bounded and analytic on $G$. If $f(z)$ approaches the value a at $p$ as $z$ approaches $p$ from either direction on $\Gamma$, then $f(z)$ approaches $a$ as $z$ tends to $p$ through $G \cup \Gamma-\{p\}$.

Recall that a sequence $\left\{z_{n}\right\}$ in $D$ is a Blaschke sequence if and only if $\sum 1-\left|z_{n}\right|$ converges. With this terminology and the above two theorems we prove

Received by the editors January $1,1967$.

1 The author was supported in part by Grant NSF GP-6118 of the National Science Foundation. 
Theorem 1. Let $K$ be a compact subset of $D \cup \Gamma$ such that $K \cap \Gamma$ $=\left\{e^{i \theta}\right\}$. Then there exists a Blaschke sequence $\left\{z_{n}\right\}$ in $D, z_{n} \rightarrow e^{i \theta}$ with the property that whenever $f \in H^{\infty}$ and $f\left(z_{n}\right) \rightarrow 0$, then

$$
\lim _{z \rightarrow e_{i \theta ; z} \in K} f(z)=0 \text {. }
$$

Proof. We may assume $e^{i \theta}=1$. Let $K^{\prime}$ be the convex hull of $K \cup \bar{K}$, where $\bar{K}$ is the set of conjugates of the points of $K$. Let $\gamma^{\prime}$ be the boundary of $K^{\prime}$. By Lindelof's Theorem to prove the present theorem it is enough to construct a Blaschke sequence $\left\{z_{n}\right\}$ on $\gamma^{\prime}$ with the property that if $f \in H^{\infty}$ and $f\left(z_{n}\right) \rightarrow 0$, then $f(z) \rightarrow 0$ as $z \rightarrow 1$ on $\gamma^{\prime}$. Now, the union of two Blaschke sequences tending to 1 is again a Blaschke sequence tending to 1 and $\gamma^{\prime}$ is symmetric about the real axis. So it is sufficient to find a sequence $\left\{z_{n}\right\}$ on the part, $\gamma$, of $\gamma^{\prime}$ terminating at 1 which lies above the real axis such that $f\left(z_{n}\right) \rightarrow 0$ implies $f(z) \rightarrow 0$ as $z \rightarrow 1$ along $\gamma$. We will use these additional properties of $\gamma: \gamma$ is convex -hence, rectifiable; as $z$ proceeds along $\gamma$ to $1,|z|$ and $\operatorname{Re}(z)$ increase monotonely to 1 . Denote the Euclidean arclength measured along $\gamma$ between $z, w \in \gamma$ by $\gamma(z, w)$.

With these preliminaries we proceed with the construction of $\left\{z_{n}\right\}$. Choose a point $w_{1}$ on $\gamma$. Let $w_{2}$ be that point on $\gamma$ satisfying $\left|w_{2}\right|>\left|w_{1}\right|$ and $\gamma\left(w_{1}, w_{2}\right)=1-\left|w_{2}\right|$. There is such a point since the length of $\gamma$ exceeds $1-\left|w_{1}\right|$. This same procedure continued indefinitely from $w_{2}$ by induction yields a sequence $\left\{w_{n}\right\}$ on $\gamma$ such that $\gamma\left(w_{n}, w_{n+1}\right)=1-\left|w_{n+1}\right|$ and $\left|w_{n}\right| \rightarrow 1$. The latter follows since $\sum_{n=1}^{\infty}\left(1-\left|w_{n+1}\right|\right)=\gamma\left(w_{1}, 1\right)<\infty$, and this also proves that $\left\{w_{n}\right\}$ is a Blaschke sequence. It is easy to find a sequence $\left\{N_{n}\right\}$ of integers with $N_{n} \rightarrow \infty$ while $\sum_{n=1}^{\infty} N_{n}\left(1-\left|w_{n+1}\right|\right)$ is still convergent. Construct a new Blaschke sequence $\left\{z_{k}\right\}$ on $\gamma$ consisting for each $n$ of the points $w_{n}=w_{n, 0}, w_{n, 1}, \cdots, w_{n, N_{n}}=w_{n+1}$, where $\gamma\left(w_{n, j}, w_{n, j+1}\right)=$ $N_{n}^{-1} \gamma\left(w_{n}, w_{n+1}\right), j=0, \cdots, N_{n}-1$. Let $z$ be any point on $\gamma$ between $w_{n, j}$ and $w_{n, j+1}$ inclusive. Then

$$
\begin{aligned}
N_{n}\left|z-w_{n, j+1}\right| & \leqq N_{n} \gamma\left(z, w_{n, j+1}\right) \leqq N_{n} \gamma\left(w_{n, j}, w_{n, j+1}\right) \\
& =\gamma\left(w_{n}, w_{n+1}\right)=1-\left|w_{n+1}\right| \leqq 1-\left|w_{n, j+1}\right| .
\end{aligned}
$$

Thus,

$$
\chi\left(z, w_{n, j+1}\right) \leqq \frac{\left|z-w_{n, j+1}\right|}{1-\left|w_{n, j+1}\right|} \leqq \frac{1}{N_{n}} .
$$

Now, suppose $f \in H^{\infty}, f\left(z_{k}\right) \rightarrow 0$, and, without loss of generality, that $|f|$ is bounded by 1 . Then, by Pick's Theorem and the last inequality 


$$
\chi\left(f(z), f\left(w_{n, j+1}\right)\right) \leqq 1 / N_{n} \rightarrow 0 .
$$

Since $f\left(w_{n, j+1}\right) \rightarrow 0, f(z) \rightarrow 0$ as $z \rightarrow 1, z \in \gamma$. This completes the proof.

In particular, the Blaschke product with zeros $\left\{z_{n}\right\}$ tends to zero as $z \rightarrow e^{i \theta}$ through $K$. By definition a function $f \in H^{\infty}$ has $T_{\mu}$-limit zero at $1, \mu \geqq 1$, in case $f(z) \rightarrow 0$ as $z \rightarrow 1$ through the sets

$$
K(m, \mu)=\left\{z: 1-|z| \geqq m(\arg z)^{\mu}, 0<|z|<1\right\}
$$

for each $m>0$. Thus, it is clear how Somadasa's Theorem may be obtained from Theorem 1.

Theorem 1 also points out that the approaches to 1 through the sets $K(m, \mu), m>0, \mu \geqq 1$, fall rather short of exhausting the different possible tangential approaches to 1 . For let $\gamma$ be a convex curve in $D \cup \Gamma, \gamma \cap \Gamma=1$, which is symmetric about the real axis. Then, from the proof of Theorem 1, there is a Blaschke product, $B(z)$, whose zeros are on $\gamma$ and which tends to zero as $z \rightarrow 1$ inside the curve $\gamma$. Furthermore, as is well known (e.g., see [2, p. 35]) $B(z)$ tends to each number of modulus not exceeding 1 on some sequence $\left\{z_{n}\right\}$ tending to 1. Each of the sequences must approach 1 more tangentially than the curve $\gamma$. This situation persists independent of how large an order of contact at 1 is chosen for $\gamma$.

\section{REFERENCES}

1. R. Nevanlinna, Eindeutige analytische Funktionen, 2nd. ed., Springer-Verlag, Berlin, 1953.

2. K. Noshiro, Cluster sets, Springer-Verlag, Berlin, 1960.

3. H. Somadasa, Blaschke products with zero tangential limits, J. London Math. Soc. 41 (1966), 293-303.

4. M. L. Weiss, "Some separation properties in sup-norm algebras of continuous functions," pp. 93-97, in Function algebras, Scott, Foresman and Co., Glenview, Ill., 1966.

University of California, Santa Barbara 